\title{
Evaluation of treatments for claw horn lesions in dairy cows in a randomized controlled trial
}

\author{
H. J. Thomas, ${ }^{* 1}$ G. G. Miguel-Pacheco, ${ }^{*}$ N. J. Bollard, ${ }^{*}$ S. C. Archer, ${ }^{*}$ N. J. Bell,† C. Mason,‡ O. J. R. Maxwell, ${ }^{*}$ \\ J. G. Remnant, ${ }^{*}$ P. Sleeman, ${ }^{*}$ H. R. Whay, $§$ and J. N. Huxley* \\ *School of Veterinary Medicine and Science, University of Nottingham, Sutton Bonington Campus, Sutton Bonington, Leicestershire, \\ LE12 5RD, United Kingdom \\ †Royal Veterinary College, Hawkshead Lane, North Mymms, Hertfordshire, AL9 7TA, United Kingdom \\ †Scotland's Rural College (SRUC), Kings Buildings, West Mains Road, Edinburgh, EH9 3JG, United Kingdom \\ $\S S c h o o l$ of Veterinary Sciences, University of Bristol, Langford House, Langford, BS40 5DU, United Kingdom
}

\begin{abstract}
Lameness is one of the most significant endemic disease problems facing the dairy industry. Claw horn lesions (principally sole hemorrhage, sole ulcer, and white line disease) are some of the most prevalent conditions. Despite the fact that thousands of animals are treated for these conditions every year, experimental evidence is limited on the most effective treatment protocols. A randomized, positively controlled clinical trial was conducted to test the recovery of newly lame cows with claw horn lesions. Animals on 5 farms were locomotion scored every 2 wk. Cows were eligible for recruitment if they had 2 nonlame scores followed by a lame score and had a claw horn lesion on a single claw of a single foot. Following a therapeutic trim, enrolled cows were randomly allocated to 1 of 4 treatments: treatment $1-$ no further treatment (positive control; TRM), treatment 2-trim plus a block on the sound claw (TB), treatment 3-trim plus a 3-d course of the nonsteroidal anti-inflammatory drug (NSAID) ketoprofen (TN), treatment 4 - trim plus a block plus ketoprofen (TBN). The primary outcome measure was locomotion score 35 d after treatment, by an observer blind to treatment group. Descriptive statistics suggested that treatment groups were balanced at the time of enrollment, that is, randomization was successful. Based on a sound locomotion score (score 0) $35 \mathrm{~d}$ after treatment, the number of cures was 11 of $45(24.4 \%)$ for TRM, 14 of $39(35.9 \%)$ for TB, 12 of $42(28.6 \%)$ for TN, and 23 of 41 (56.1\%) for TBN. The difference between TBN and TRM was significant. To test for confounding imbalances between treatment groups, logistic regression models were built with 2 outcomes, either sound (score 0) or nonlame
\end{abstract}

Received October 16, 2014.

Accepted February 13, 2015.

${ }^{1}$ Corresponding author: stxht1@nottingham.ac.uk (score 0 or 1) $35 \mathrm{~d}$ after treatment. Compared with TRM, animals that received TBN were significantly more likely to cure to a sound outcome. Farm, treatment season, lesion diagnosis, limb affected, treatment operator, and stage of lactation were included in the final models. Our work suggests that lameness cure is maximized with NSAID treatment in addition to the common practices of therapeutic trimming and elevation of the diseased claw using a block when cows are newly and predominantly mildly lame.

Key words: dairy cow, lameness, claw horn lesion, randomized clinical trial

\section{INTRODUCTION}

Lameness in dairy cattle is a significant problem in intensive dairy industries around the world, causing production losses (Huxley, 2013) and discomfort, undermining animal welfare (Whay et al., 1997). Achieving sustainable reductions in the levels of disease on farm requires a combination of 2 approaches: first, the implementation of effective farm-specific prevention strategies to decrease the rate at which new cases develop, and second, early identification and prompt and effective treatment of clinical cases to reduce the duration of time over which animals are lame. Whereas the emphasis of the majority of recent research has rightly focused on identifying risk factors for lameness and disease prevention, the treatment of animals once they become lame must not be neglected.

Sole hemorrhage, sole ulcer, and white line disease (the most common claw horn lesions) are some of the most prevalent conditions causing lameness (Capion et al., 2008; Cramer et al., 2008). Despite the fact that many thousands of animals are routinely treated for these diseases, a recent systematic review of the peer-reviewed literature on the prevention and treatment of foot lameness in cattle highlighted the deficit 
of information in this area (Potterton et al., 2012). In literature published between 2000 and 2011, no papers were identified that were concerned with the treatment of white line disease and only 3 were concerned with the treatment of sole ulcers. Of these, 2 were case studies (i.e., not experimental), and although the third was composed of primary research, it assessed dietary supplementation with biotin (Lischer et al., 2002) and is of limited use in the field. The authors concluded that virtually all the existing information on the treatment of claw horn lesions appeared to be from anecdotal reports based on the experience and knowledge of experts working in the field. This does not mean to say that current treatment protocols are ineffective, rather it highlighted the deficit of experimental evidence on the most effective treatment, that is, those that lead to the highest cure rates in the shortest time.

An extension of the literature search described above confirms that very little primary research work has ever been published testing treatments for claw horn lesions; only 2 other peer-reviewed papers were identified. The first describes a randomized study conducted in Australia that tested wooden blocks, rubberized shoes, and padded bandages containing copper sulfate for the treatment of a variety of claw horn lesions (Pyman, 1997). Three and $7 \mathrm{~d}$ after treatment, a significantly high number of cows had recovered in the block and shoe groups compared with the bandage group; outcome assessment was limited to $14 \mathrm{~d}$ after treatment by which time no differences between groups were apparent. In the second, dairy cows managed under New Zealand's extensive pasture-based systems were randomly treated with a plastic shoe and the nonsteroidal anti-inflammatory drug (NSAID) tolfenamic acid, following corrective trimming (Laven et al., 2008). The authors concluded treatments did not significantly differ in either nociceptive threshold or locomotion score over the 100-d outcome period. The objective of the present study was to compare 4 treatments for claw horn lesions in a randomized study under UK field conditions.

\section{MATERIALS AND METHODS}

\section{Study Design and Reporting}

A positively controlled, randomized clinical trial (RCT) with blind outcome observations was designed to test the recovery of dairy cows with claw horn lesions, treated using different protocols. The study hypothesis stated that the likelihood of claw-horn-lesion recovery depended on the treatment administered. Based on a binary primary outcome measure (lame or not lame) after treatment, a power calculation suggested that treat- ment group sizes of 58 would detect a $25 \%$ difference in recovery rate between treatments (power value of 0.8 , $P \leq 0.05)$. A difference of $25 \%$ was selected because it was considered clinically meaningful and likely to be large enough to warrant the additional cost of the treatments tested should they prove superior.

The study was positively controlled (i.e., no animals were left untreated) and conducted under the Veterinary Surgeons Act 1966, which regulates acts of veterinary surgery in the UK. The protocol was reviewed and approved by the University of Nottingham's School of Veterinary Medicine and Science Ethical Review Committee before study instigation.

The study manuscript has been prepared in accordance with the guidelines outlined in the REFLECT statement for reporting randomized controlled trials in livestock (O'Connor et al., 2010).

\section{Herd Selection}

A convenience sample of 5 commercial dairy farms was recruited in the East Midlands area of the UK, within close proximity to the University of Nottingham. To be eligible for enrollment, farms were required to have a herd lameness prevalence of above $20 \%$ at the start of the study and be undertaking routine measures to control digital dermatitis at the herd level (e.g., regular foot bathing). Farms were either known to the trial coordinators or were recruited through their veterinary surgeons, who were asked to nominate clients they considered met the criteria and would be willing to participate. A short list of suggested farms were approached and visited to discuss the trial and to assess their lameness prevalence. Following an introductory phone call, one farm elected not to participate because they thought the trial would interfere with their day-today farm management.

The 5 farms were between 187 and 353 (median 241) cows in size with 305-d adjusted milk yields ranging from 7,394 to $11,579 \mathrm{~L}$ (median $=10,381 \mathrm{~L}$ ). Three of the farms (farms 2, 4, and 5) housed lactating cows continuously; the other 2 farms managed cows at pasture during the summer ( $\sim$ March-October) and in housing during winter. On all farms, lactating cows were accommodated in stalls with mats, mattresses, or waterbeds. Two farms (farms 2 and 4) milked cows in an automatic milking system; the remaining farms milked cows in conventional parlors, 2 times daily. All walkways and standing areas were concrete on all farms except farm 2, which had rubber matting throughout, and farm 3, which had rubber matting at the feed face of the high-yielding group. All farms undertook routine foot trimming, although scheduling ranged from as required to weekly sessions. Two farms (farms 1 and 2) 
used an external professional foot trimmer, and on the other farms, trimming was conducted by farm staff. All the farm routines were that lame cows were treated as soon as they were identified or at weekly or fortnightly routine health sessions, depending on disease severity and staff availability. Farmers were advised to continue their normal procedures for identifying and treating lame cows throughout the study period.

\section{Cow Selection and Enrollment Criteria}

Beginning in December 2011, locomotion scoring of all cows in the lactating herd was undertaken at fortnightly intervals, by trained experienced observers $(H$. T., G. M. P., N. J. B. ), as cows exited the milking parlor (farms 1, 3, and 5) or in a passageway with a firm, level surface (farms 2 and 4). All animals in all herds were uniquely identified by freeze brand, which was used to distinguish individual cows. Dry cows and young stock were not scored. Cows were scored on a 6 -point scale adapted from the Great Britain industry standard scoring system (Table 1); for animals considered lame $(>1)$, the lame limb was identified and recorded.

Animals were considered for enrollment if they presented with a new case of lameness in a single hind limb, that is, 2 successive nonlame scores ( 0 or 1$)$ followed by a lame score $(>1)$. Animals were excluded if they had received treatment for lameness in the same foot within $120 \mathrm{~d}$ or treatment for lameness in another foot within $90 \mathrm{~d}$ or had completed a course of parenteral antibiotics or NSAID within the previous $14 \mathrm{~d}$.

Selected cows were examined within $48 \mathrm{~h}$ of the locomotion scoring. Animals were assessed for BCS according to Edmonson et al. (1989) using a scale of 1 to 5 with increments of 0.5 . The lame foot was inspected with the animal restrained in a foot-trimming crush. Animals were excluded if they were diagnosed with interdigital necrobacillosis, active digital dermatitis (an M1, M2, or M4.1 lesion; Berry et al., 2012), substantial interdigital hyperplasia, or a significant hock lesion. Identification of the painful claw was attempted by lateral rotation of the claw resulting in a withdrawal reflex and the application of hoof testers. Each animal received a therapeutic trim of the whole foot (i.e., both claws) consisting of a standard trim, investigation and trimming out of any lesions identified, removal of diseased and underrun horn, and rebalancing of the claw height to reduce weight bearing on the diseased claw (Toussaint Raven, 2002). Animals were excluded from the study when lesions were identified in both claws, that is, only animals with a claw horn lesion on one claw of a single lame hind leg were eligible for inclusion.

Animals that did not meet these enrollment criteria were treated but not enrolled. They took no further part in the study, but they could be considered again in the future provided the minimum lag periods since treatment had elapsed. Animals could only be enrolled in the study once; if they presented with lameness on the same or a different leg in the future, they were excluded.

\section{Lesion Classification}

Claw lesions identified during examination of the feet of enrolled animals were classified into 1 of 3 groups:

1. sole hemorrhage or sole ulceration (SHU): lesion or lesions composed of hemorrhage or an ulcer of the sole in any location;

2. white line disease (WLD): lesion or lesions of any severity (hemorrhage through to complete separation) at any location on the white line; and

Table 1. Locomotion scoring descriptors employed in a randomized clinical trial to test the recovery of dairy cows from claw horn lesions

\begin{tabular}{ll}
\hline $\begin{array}{l}\text { Locomotion } \\
\text { score }^{1}\end{array}$ & Descriptor \\
\hline 0 & $\begin{array}{l}\text { Walks with even weight bearing and rhythm on all } 4 \text { feet, with a flat back. Long fluid strides possible. } \\
1\end{array}$ \\
Steps uneven (rhythm or weight bearing or strides shortened, affected limb or limbs not immediately identifiable). \\
Mild asymmetry in hind-limb movement. Decreased stride length on affected limb and slightly decreased stance duration \\
with a corresponding increase in limb flight velocity on the nonaffected side. Walking velocity remains normal. Back may \\
be raised. \\
Moderate asymmetry in hind-limb movement. Decreased stride length on affected limb and a distinct decrease in stance \\
duration. Limb flight on the nonaffected limb is correspondingly faster and the overall walking velocity is reduced. Back \\
usually raised. \\
Severe asymmetry in hind-limb movement. Marked decrease in stride length on affected limb and very short stance \\
duration. Limb flight on nonaffected limb rapid and walking velocity reduced such that cow cannot keep up with healthy \\
herd. Back raised.
\end{tabular}

${ }^{1}$ Adapted, with permission, from the DairyCo Mobility Score system, the Great Britain industry standard. Scores 2a and 2b and 3a and 3b can be amalgamated back to scores 2 and 3 in this system, respectively. 
3. other claw horn lesion: any other claw horn lesion that could not be categorized as SHU or WLD or 2 or more different lesions on the same claw (e.g., SHU and WLD).

\section{Randomization and Treatments Administered}

Enrolled animals were randomly allocated to 1 of 4 treatment groups (Table 2) using a computer-generated randomization plan (www.randomization.com, work conducted by H. T.) created in blocks of 4, with each of the 4 treatment groups included once in each block. Randomization was further blocked by farm and lesion type (SHU, WLD, or other), to ensure approximate temporal matching of equal numbers of cows with each diagnosis within each study farm. Group 1 (therapeutic trim only; TRM) was considered the positive control group. Following completion of the therapeutic trim, animals were allocated to treatment group by drawing from a card index box consecutively numbered cards that had the treatment written on the reverse side.

Drawing of the randomization cards and administration of treatments were conducted by trained veterinary surgeons familiar with the treatment of lame cows and predominantly undertaken by a single operator $(\mathrm{H}$. T.) with vacation cover (S. A., O. M., J. H., and J. R.). Operators administering treatments were not blind to the treatment administered. Enrolled animals were identified with a leg band on both hind limbs. Farmers were asked to continue managing them in accordance with normal farm management practices but were requested not to treat them for lameness and to notify the researcher if they felt that further intervention was necessary. Farmers were not blind to treatment group; although they were not provided with a list of treatments administered, the presence of therapeutic blocks could be observed and treatment with NSAID was recorded in their medicine records.

\section{Treatment Follow-Up and Outcome Observations}

Animals were reexamined $8 \mathrm{~d}( \pm 3 \mathrm{~d})$ after treatment. If a foot block had been applied as part of the treatment protocol [treatment 2 (TB) and treatment 4 $(\mathrm{TBN})]$ and it was no longer present, it was reapplied. If locomotion score had deteriorated from that at the time of enrollment, animals were re-treated.

Animals in groups TB and TBN were reexamined for a second time, $28 \mathrm{~d}( \pm 3 \mathrm{~d})$ after treatment. If the block was still present, it was manually removed using trimming pincers and careful leverage. This was the only action undertaken at this time point, that is, no additional treatments were administered.
The primary outcome measure, locomotion score 35 $\mathrm{d}( \pm 4 \mathrm{~d})$ after treatment, was conducted by an independent observer (G. M. P.) blind to treatment group. That observer collected outcome scores with cows walking in isolation, on a firm, level surface. For animals considered lame $(>1)$, the lame limb was identified and recorded. Following the blind outcome score, animals were body-condition scored using the method previously described, and the treated limb was elevated and examined for digital dermatitis and any other conditions.

\section{Additional Data Collected}

Data on parity, monthly milk yield, and calving date were collated from farm records. Animals that were sold, culled, or died before assessment of the primary outcome measure were recorded and withdrawn from the study.

\section{Data Collation and Statistical Analysis}

Data collected for each cow at each visit were recorded onto data-capture forms and then transcribed and stored in a relational database (Access 2007, Microsoft Corporation, Redmond, WA). Data analysis was conducted in Minitab 16 (Minitab Inc., State College, PA). Data were audited for validity and spurious records using entry rules set up in the database and by manually scanning for outlying data following sorting within each data category. For analysis, locomotion scores $2 \mathrm{a}$ and $2 \mathrm{~b}$ and $3 \mathrm{a}$ and $3 \mathrm{~b}$ were amalgamated to 2 and 3 , respectively.

Differences between treatment groups at the time of enrollment were assessed by ANOVA (DIM and last recorded monthly yield) and using the Kruskal-Wallis test (lameness score at treatment, BCS at treatment and parity).

A successful treatment at study outcome (35 d after treatment) was defined as either (1) a sound locomotion score (score 0 ) or (2) a nonlame score (score 0 or 1 ). The proportions of successful treatments in animals that received TB, TN (treatment group 3), and TBN were each compared with TRM using the $\chi^{2}$ test. A Bonferroni corrected $P$-value was calculated to account for multiple comparisons; the significance probability was set at $P \leq 0.05$ for a 2 -tailed test.

To test for confounding effects in the results, a multivariable analysis was conducted. Logistic regression models were built in MLwiN (Version 2.1, Centre for Multilevel Modeling, University of Bristol, Bristol, UK), with the same outcomes described above: (1) a sound locomotion score (score 0) $35 \mathrm{~d}$ after treatment and (2) 
Table 2. Treatment administered in a randomized clinical trial designed to test the recovery of dairy cows from claw horn lesions

\begin{tabular}{|c|c|c|}
\hline $\begin{array}{l}\text { Treatment } \\
\text { group }\end{array}$ & Treatment & Description \\
\hline 1 (TRM) & $\begin{array}{l}\text { Therapeutic trim only } \\
\text { (positive control group) }\end{array}$ & 1. Therapeutic trim applicable to the lesion \\
\hline $3(\mathrm{TN})$ & $\begin{array}{l}\text { Therapeutic trim plus } \\
\text { NSAID }^{2}\end{array}$ & $\begin{array}{l}\text { 1. Therapeutic trim applicable to the lesion } \\
\text { 2. Administration of a } 3 \text {-d course of ketoprofen (Ketodale } 100 \mathrm{mg} / \mathrm{mL} \text {, Richter Pharma AG) } \\
\text { administered by deep intramuscular injection at } 3 \mathrm{mg} \text { of ketoprofen per kilogram of BW }\end{array}$ \\
\hline
\end{tabular}

${ }^{1}$ Approximately $110 \mathrm{~mm}$ long, $55 \mathrm{~mm}$ wide, and $23 \mathrm{~mm}$ deep. The block was positioned based on the experience of the worker in an attempt to replicate normal claw placement and weight distribution. Where necessary the block was positioned toward the heel (away from the toe) to ensure weight was borne on the flat of the block. Demotec, Nidderau Germany; Richter Pharma AG, Wels, Austria.

${ }^{2} \mathrm{NSAID}=$ nonsteroidal anti-inflammatory drug.

a nonlame score (score 0 or 1) $35 \mathrm{~d}$ after treatment. Farm and treatment were forced into the models as categorical fixed effects. Other variables and plausible interactions were investigated by forward selection, for inclusion stepwise. Variables were eliminated from the model based on the Wald test if $P \leq 0.05$. Variables tested included parity $(1,2,3, \geq 4)$, DIM, calving season (winter, spring, summer, autumn), season of treatment, locomotion score at treatment, lame leg at treatment, BCS at treatment and outcome, lesion classification (SHU, WLD, other), active digital dermatitis at outcome (yes or no), re-treatment required at 8-d recheck visit (yes or no), reapplication of block required at 8-d recheck visit (yes or no), treatment operator [principal operator (H. T.) or other operators (S. A., O. M., J. H., J. R.)], and milk yield at the last 2 monthly recordings. Days in milk was tested as a linear mean centered variable, a categorical variable in $30-d$ increments, and as a nonlinear variable, $\mathrm{e}^{(-0.065 \times \mathrm{DIM})}$ (Silvestre et al., 2006).

To assess fit, model predictions were compared with the observed data in groups stratified by categorical variables in the model, such as treatment group. Predictions were generated by simulation. The models were deemed adequate if observed values were within 95\% confidence intervals of prediction.

\section{RESULTS}

\section{Study Inclusions}

Between January 10, 2012, and January 31, 2013, a total of 512 cows met the initial selection criteria and were examined. Enrollment of cows on farm 3 was suspended on April 24, 2012, because of the very low numbers of animals that were becoming eligible for enrollment (i.e., the number of new cases of lameness had dropped substantially from the start of the study).
Farm 5 was recruited as a replacement; enrollment began on July 17, 2012, and continued to the end of the study. Selection of cows on farm 3 recommenced on November 16, 2012, and continued to the end of the study. Of the selected and examined cows, 183 met all of the inclusion criteria and were enrolled into the RCT. The remaining 329 animals were not enrolled for the following reasons: $227(68.9 \%)$ had a lesion on both claws; $27(8.2 \%)$ had no visible lesion on either claw and no painful claw could be identified; $2(0.6 \%)$ were no longer lame, $41(12.5 \%)$ had active digital dermatitis, $3(0.9 \%)$ had interdigital necrobacillosis, $1(0.3 \%)$ had an interdigital hyperplasia, $6(1.8 \%)$ had a hock lesion, $14(4.3 \%)$ had been treated by farm staff, and $8(2.4 \%)$ were not compliant with the study protocol.

The number of cows allocated to each of the treatment groups by lesion diagnosis and farm is outlined in Table 3. In total 47 cows received TRM, 46 TB, 45 TN, and 45 TBN. Of the enrolled cows, $171(93.4 \%)$ presented with a locomotion score of 2 and $12(6.6 \%)$ with a score of 3 .

\section{Study Exclusions}

Sixteen enrolled cows were withdrawn before the primary outcome was assessed. One animal (farm 1, TN) was culled; 5 animals (farm 2 , TB $\times 2$; farm 4 , TBN $\times$ 1 ; farm $5, \mathrm{~TB} \times 1$ and $\mathrm{TBN} \times 1$ ) were withdrawn for noncompliance with the study protocol after enrollment (e.g., becoming unduly stressed or repeated collapsing in the crush); 4 animals (farm $2, \mathrm{TN} \times 1$ and TBN $\times 1$; farm $4, \mathrm{~TB} \times 1$ and TBN $\times 1$ ) were re-treated by the farmer without informing the researcher, and 6 animals (farm $1, \mathrm{~TB} \times 1$ and $\mathrm{TN} \times 1$; farm $2, \mathrm{~TB} \times 1$; farm 4 , $\mathrm{TRM} \times 1$ and $\mathrm{TN} \times 1$; farm $5, \mathrm{TRM} \times 1$ ) were lost to the study or were unavailable for reassessment for other 
Table 3. Number of cows allocated to each of 4 treatment groups ${ }^{1}$ by lesion diagnosis and farm in a randomized clinical trial designed to test the recovery of dairy cows from claw horn lesions

\begin{tabular}{|c|c|c|c|c|c|c|c|c|c|c|c|c|c|}
\hline \multirow{2}{*}{ Item } & \multicolumn{12}{|c|}{ Lesion diagnosis } & \multirow{2}{*}{ Total } \\
\hline & \multicolumn{4}{|c|}{ Sole hemorrhage or ulcer } & \multicolumn{4}{|c|}{ White line disease } & \multicolumn{4}{|c|}{ Other lesion ${ }^{2}$} & \\
\hline \multicolumn{14}{|l|}{ Farm ID } \\
\hline 1 & 6 & 6 & 5 & 5 & 1 & 1 & 1 & 1 & 3 & 4 & 3 & 3 & 39 \\
\hline 2 & 4 & 5 & 5 & 5 & 4 & 4 & 3 & 4 & 3 & 2 & 3 & 3 & 45 \\
\hline 5 & 2 & 2 & 2 & 1 & 1 & 0 & 1 & 1 & 4 & 4 & 4 & 4 & 26 \\
\hline Total & 21 & 21 & 21 & 20 & 10 & 9 & 8 & 9 & 16 & 16 & 16 & 16 & 183 \\
\hline Grand total & \multicolumn{4}{|c|}{83} & \multicolumn{4}{|c|}{36} & \multicolumn{4}{|c|}{64} & \\
\hline
\end{tabular}

${ }^{1} \mathrm{TRM}=$ therapeutic trim only; $\mathrm{TB}=$ therapeutic trim plus block on the sound claw; $\mathrm{TN}=$ therapeutic trim plus 3 -d course of nonsteroidal anti-inflammatory drug (NSAID); TBN = therapeutic trim plus block plus NSAID.

${ }^{2}$ Predominantly a combination of both sole hemorrhage or ulcer and white line disease.

reasons (e.g., moved to a distant location or incorrectly identified). Of the remaining 167 enrolled animals, 6 animals (farm 1 , TRM $\times 1$ and TB $\times 1$; farm 2 , TB $\times$ 1 and $\mathrm{TN} \times 1$; farm $4, \mathrm{~TB} \times 1$ and $\mathrm{TBN} \times 1$ ) required re-treatment at the 8 -d recheck visit. Two received additional trimming, 2 had their foot block removed and repositioned, 1 was treated for digital dermatitis with topical oxytetracyline spray (Alamycin aerosol 3.58\% wt/wt cutaneous spray solution, Norbrook, Newry, Northern Ireland), and 1 received treatment for a hock lesion by cleaning and the application of topical oxytetracyline spray. Seventeen animals that received TB (7 animals) and TBN (10 animals) required the reapplication of a foot block at the 8-d recheck visit because it was no longer present. A total of 144 cows were treated by the principal operator (H. T.), and 23 cows were treated by other operators (S. A., J. R., J. H. or O. M.).

\section{Descriptive Results and Univariate Analysis}

The parity, DIM, last recorded milk yield, and BCS and lameness score at treatment of enrolled cows by treatment group are outlined in Table 4. Differences between groups were not significant.
The locomotion scores of enrolled cows at outcome, $35 \mathrm{~d}$ after treatment, are outlined in Table 5 . Based on a sound score (score 0), the number (and percentage) of successful treatments was 11 of $45(24.4 \%)$ for TRM, 14 of $39(35.9 \%)$ for TB, 12 of $42(28.6 \%)$ for TN, and 23 of $41(56.1 \%)$ for TBN. The difference between TBN and TRM was significant (Bonferroni corrected $P=$ $0.01)$.

Based on a nonlame score (score 0 or 1 ), the number (and proportion) of successful treatment was 31 of 45 $(68.8 \%)$ for TRM, 28 of $39(71.8 \%)$ for TB, 32 of 42 $(76.2 \%)$ for $\mathrm{TN}$, and 35 of $41(85.3 \%)$ for TBN. The differences between groups were not significant.

Of the lame animals $35 \mathrm{~d}$ after treatment, the number (and proportion) of animals lame on the leg that was treated at enrollment was 8 of $14(57.1 \%)$ for TRM, 4 of $11(36.4 \%)$ for TB, 5 of $10(50 \%)$ for TN, and 5 of 6 $(83.3 \%)$ for TBN.

\section{Logistic Regression Analysis}

Of the enrolled cows, 85 and 66 had missing milk recording records in the preceding 1 and $2 \mathrm{mo}$, respec-

Table 4. Descriptive statistics of animals in each of 4 treatment groups ${ }^{1}$ in a randomized clinical trial designed to test the recovery of dairy cows from claw horn lesions

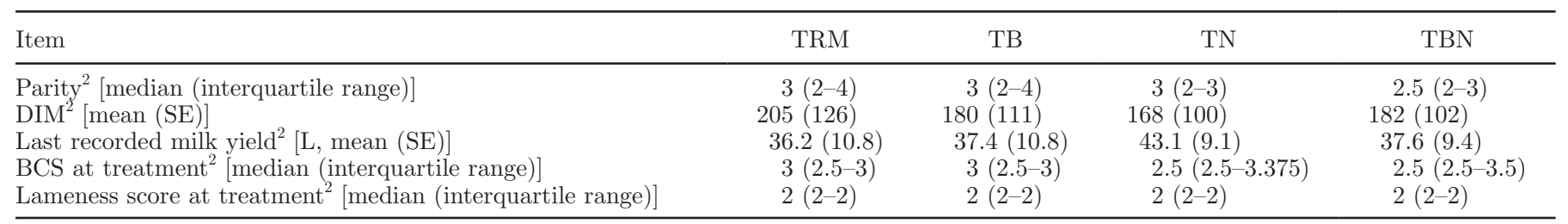

${ }^{1} \mathrm{TRM}=$ therapeutic trim only; $\mathrm{TB}=$ therapeutic trim plus block on the sound claw; $\mathrm{TN}=$ therapeutic trim plus 3-d course of nonsteroidal anti-inflammatory drug (NSAID); TBN = therapeutic trim plus block plus NSAID.

${ }^{2}$ Differences between treatment groups were not significant. 
Table 5. Locomotion score $35 \mathrm{~d}$ after treatment in dairy cows recruited to a randomized clinical trial designed to test recovery from claw horn lesions

\begin{tabular}{lcccc}
\hline & \multicolumn{4}{c}{ Locomotion score 35 d after treatment [score (\%)] } \\
\cline { 2 - 5 } Treatment $^{1}$ & $0^{2}$ & $1^{2}$ & 2 & 3 \\
\hline TRM $(\mathrm{n}=45)$ & $11(24.4)$ & $20(44.4)$ & $14(31.1)$ & 0 \\
TB $(\mathrm{n}=39)$ & $14(35.9)$ & $14(35.9)$ & $10(25.6)$ & $1(2.6)$ \\
TN $(\mathrm{n}=42)$ & $12(28.6)$ & $20(47.6)$ & $10(23.8)$ & 0 \\
TBN $(\mathrm{n}=41)$ & $23(56.1)$ & $12(29.3)$ & $6(14.6)$ & 0 \\
\hline
\end{tabular}

${ }^{1} \mathrm{TRM}=$ therapeutic trim only; $\mathrm{TB}=$ therapeutic trim plus block on the sound claw; $\mathrm{TN}=$ therapeutic trim plus 3-d course of nonsteroidal anti-inflammatory drug (NSAID); TBN = therapeutic trim plus block plus NSAID.

${ }^{2}$ Score $0=$ sound; scores 0 and $1=$ nonlame.

tively. Milk recording records in the 2 mo preceding treatment were tested in models based on subsets of the data set with no missing records. Eight animals had missing records for DIM and were discarded.

Model fit to the data was acceptable, and results of the logistic regression models are outlined in Table 6 . In the first model testing cure to outcome (1) (score 0 ), animals in the TBN group were significantly more likely to cure compared with cows in the TRM group
$(P \leq 0.05)$. Cows treated on farm 5 , compared with other study farms, and treatments in spring and autumn, compared with treatments in winter, were less likely to cure.

In the second model testing cure to outcome (2) (score 0 or 1), treatment group was not significant; however, there was a trend for animals in the TBN group to be more likely to cure compared with cows in the TRM group (odds ratio $=3.2,95 \% \mathrm{CI}=0.9-11.3$ )

Table 6. Outcomes from logistic regression models in a randomized clinical trial designed to test the recovery of dairy cows from claw horn lesions (odds ratio scale unless shown otherwise)

\begin{tabular}{|c|c|c|c|c|c|c|}
\hline \multirow[b]{3}{*}{ Model term ${ }^{1}$} & \multicolumn{3}{|c|}{$\begin{array}{l}\text { Outcome } 1 \text {. Sound locomotion } \\
\text { score } \\
\text { (score 0) } 35 \mathrm{~d} \text { after treatment }\end{array}$} & \multicolumn{3}{|c|}{$\begin{array}{l}\text { Outcome } 2 . \text { Nonlame locomotion } \\
\text { score } \\
\text { (score } 0 \text { or } 1 \text { ) } 35 \mathrm{~d} \text { after treatment }\end{array}$} \\
\hline & \multirow[b]{2}{*}{ Odds ratio } & \multicolumn{2}{|c|}{$95 \% \mathrm{CI}$} & \multirow[b]{2}{*}{ Odds ratio } & \multicolumn{2}{|c|}{$95 \% \mathrm{CI}$} \\
\hline & & $2.5 \%$ & $97.5 \%$ & & $2.5 \%$ & $97.5 \%$ \\
\hline Intercept & $\begin{array}{c}-1.08 \\
\text { Reference }\end{array}$ & -2.14 & -0.05 & 3.28 & 0.82 & 13.1 \\
\hline TB & $\begin{array}{l}\text { Keference } \\
2.1\end{array}$ & 0.8 & 5.8 & $\begin{array}{l}\text { Reference } \\
\quad 1.2\end{array}$ & 0.4 & 3.8 \\
\hline $\mathrm{TN}$ & 1.2 & 0.4 & 3.2 & 1.3 & 0.4 & 4.3 \\
\hline TBN & $6.4^{*}$ & 2.4 & 18.0 & 3.2 & 0.9 & 11.3 \\
\hline Farm 1 & Reference & & & Reference & & \\
\hline Farm 2 & 0.6 & 0.2 & 1.8 & 0.7 & 0.2 & 2.5 \\
\hline Farm 3 & 1.3 & 0.4 & 4.3 & 3.6 & 0.6 & 21.9 \\
\hline Farm 4 & 1.2 & 0.5 & 3.5 & 1.1 & 0.3 & 4.0 \\
\hline Farm 5 & $0.1^{*}$ & 0.0 & 0.4 & 0.7 & 0.2 & 2.8 \\
\hline Right hind limb & Reference & & & Reference & & \\
\hline Left hind limb & $4.8^{*}$ & 2.3 & 10.5 & $2.3^{*}$ & 1.0 & 5.5 \\
\hline $\mathrm{e}^{-0.065 \times \mathrm{DIM}}($ logit scale $)$ & $8.5^{*}$ & 3.5 & 13.9 & $7.8^{*}$ & 2.3 & 13.3 \\
\hline Winter treated & Reference & & & & & \\
\hline Spring treated & $0.2^{*}$ & 0.1 & 0.4 & & & \\
\hline Summer treated & 0.4 & 0.1 & 1.1 & & & \\
\hline Autumn treated & $0.1^{*}$ & 0.0 & 0.3 & & & \\
\hline Sole ulcer or hemorrhage & & & & Reference & & \\
\hline White line disease & & & & 0.8 & 0.2 & 2.6 \\
\hline Other lesion & & & & $0.3^{*}$ & 0.1 & 0.9 \\
\hline $\begin{array}{l}\text { Principal treatment operator }(\mathrm{H} . \\
\text { T.) }\end{array}$ & & & & Reference & 0.1 & 0.8 \\
\hline Other treatment operators & & & & $0.3^{*}$ & & \\
\hline
\end{tabular}

${ }^{1} \mathrm{TRM}=$ therapeutic trim only; $\mathrm{TB}=$ therapeutic trim plus block on the sound claw; $\mathrm{TN}=$ therapeutic trim plus 3-d course of nonsteroidal anti-inflammatory drug (NSAID); TBN = therapeutic trim plus block plus NSAID; spring = March, April, and May; summer = June, July, and August; autumn = September, October, and November; winter $=$ December, January, and February.

$* P \leq 0.05$. 
Cows with other lesions had lower odds of cure compared with cows treated for SHU, and animals treated by other operators were less likely to cure than those treated by the principal operator.

In both models, animals treated on the left hind limb were more likely to cure (compared with those treated for lameness on the right hind limb), and cows were more likely to recover when treated in early lactation, with exponential decay in the relationship with time after calving.

\section{DISCUSSION}

In this study, lame cows treated for a claw horn lesion in a single claw of a single leg recovered at different rates depending on the treatment administered. Cows treated with a therapeutic trim, block, and NSAID were more likely to recover to a sound locomotion score than those treated with a therapeutic trim alone.

One of the surprising findings from our study was how small the differences in treatment success were between therapeutic trim and the application of a block to the sound claw and therapeutic trim alone. Only when an NSAID was added to the block and trim were significant differences in outcome seen. The application of a block to the sound claw as a treatment for lameness is a common practice around the world. In a recent review of textbooks and gray literature (e.g., reports and control plans; Potterton et al., 2012), 85\% of sources advocated their use for claw horn lesion. Behind a therapeutic trim, therapeutic blocks were the next most common treatment option described. Similarly, in a recent survey of UK dairy farmers, more than $90 \%$ reported using blocks and $70 \%$ considered trim and block an effective treatment for claw horn lesions (Horseman et al., 2013).

The etiology of claw horn lesions has not been fully elucidated; whatever the underlying cause, compression of the sole corium leads to vascular compromise, ischemia, hemorrhage, and ultimately interruption of keratogenesis and the development of lesions. The application of a block to the sound claw is thought to reduce load bearing and hence compression of the corium in the diseased claw and allow the compromised tissues to heal. It is noteworthy that only marginal, nonsignificant differences in cure rates were observed following the administration of NSAID without a block or a block without NSAID. This suggests that reduction in load bearing and NSAID action were synergistic in this study. We propose 2 hypotheses for this observation. First, the NSAID could be having a direct effect at the corium, reducing inflammation and assisting the corium to heal if loading is reduced by a block. Alternatively, it seems credible that blocks may cause some discomfort following application; this may modify behavior (e.g., changing lying or feeding time) or cause a redistribution of weight bearing between the claws and limbs, leading to a reduction in the rate of healing of the diseased claw. Administration of an NSAID in combination with a block may mitigate these possible changes. Our results provide some circumstantial evidence of this effect. At outcome (35 d after treatment), 6,7 , and 5 cows were lame on the contralateral hind leg in the TRM, TB, and TN groups, respectively; this compares to just 1 cow in the TBN group. Lame cows in the TRM, TB, and TN groups may have increased loading on the contralateral hind limb, predisposing it to lesion progression and lameness. Cows in the TBN group may have been comfortable to bear weight evenly on the lame limb, while at the same time the block allowed the diseased claw to heal. Further work is required to confirm our findings and better understand the mechanisms of action and benefits of different treatment options in cows with claw horn lesions.

Our results disagree with those reported by Laven et al. (2008), who saw no difference in outcomes between lame cows with claw horn lesions treated with blocks and the NSAID tolfenamic acid in addition to a therapeutic trim alone. Although the study designs are not directly comparable, they have a range of similarities making comparisons between outcomes legitimate. The differences in outcome observed could be due to differences in case selection (identified by an external observer as soon as lame vs. identified by farm staff and therefore likely to be more chronically lame), management system (more intensive predominantly housed vs. more extensive predominantly pasture based), cow type (predominantly higher yielding Holstein type vs. predominantly lower yielding Friesian and Jersey type), or other unidentified factors.

The study population recruited to this RCT was a convenience sample. That said we have no reason to suspect that it was not broadly representative of both cow and farm types common in the UK (all be it that 2 of the study farms used automatic milking systems). Enrolled cows selected from this population were predominantly newly and mildly lame. A previous study reported a median lag of $65 \mathrm{~d}$ between when cows can first be identified as lame by an external observer and when they were identified for treatment by farmers (Leach et al., 2012). This may be because, as recent work suggests, many farmers do not identify or refer to milder cases as "lame" (i.e., score 2 in this study). It appears they reserve the term "lame" for more severe cases (i.e., score 3 in this study; Horseman et al., 2014). Consequently, if farmers do not consider that milder cases are "lame," it stands to reason that they would not necessarily be considered for treatment. In our 
study, animals were locomotion scored every $2 \mathrm{wk}$ and treated as soon as they became identifiably lame. The period of time that could have elapsed between animals first becoming lame and being treated ranged between 2 and $16 \mathrm{~d}$ (fortnightly locomotion scoring plus lag to treatment visit). The majority of cows (93\%) presented with the mildest lameness classification (score 2). This population was selected first because we considered it ethically questionable to identify and then knowingly leave lame animals for several weeks before they were treated and second because we believe that these are the animals that the industry should be targeting for treatment. Readers should note that our study population, and consequently our results, may not reflect the cases that many farmers routinely identify and present for treatment, and at this stage it is not possible to say whether our results are generalizable to more severe or chronic cases managed in different farm systems. Further studies are needed to replicate this type of clinical trial to test treatment protocols in more chronically and severely lame animals, providing this work does not encourage or condone delayed treatment on farm.

A range of other variables were significant in the final models (i.e., they significantly affected cure), including farm, limb treated, DIM, season of treatment, diagnosis, and operator. Of note, cure rates to soundness on one farm (farm 5) were significantly worse than on other study farms. Despite identical case selection criteria, an unidentified factor or factors significantly affected outcome following all treatments on this unit. Clinically, it is important that farms with poor cure rates are identified and the reasons for poor responses are explored to limit the effects of this painful disease on health and welfare. It is also interesting to note that cows were more likely to recover from lameness when treated in early lactation and that the relationship exponentially decayed with time after calving. Although animals were not enrolled until at least $120 \mathrm{~d}$ had elapsed since their last treatment on the same limb, the reduction in treatment success could reflect lower recovery rates in feet with more chronic lesions from previous lameness events. Finally, the reasons for the difference in cure rates between left and right limbs is unclear; it could reflect an operator bias based on the relative ease of trimming left and right feet, depending on the dominant hand of the worker.

Logistically, this was a complex, expensive, and timeconsuming study protocol to conduct; this may explain why so few of these studies have been conducted previously. The low proportion of cows that met all the selection criteria was particularly challenging; more than 500 animals had to be examined and trimmed to enroll 183 cows. The principal reason for exclusion, making up almost $70 \%$ of exclusion, was animals having le- sions on both claws, that is, even if the claw causing the lameness was obvious, large numbers of animals had mild lesions on the contralateral claw. Although in practice, therapeutic blocks are often applied to claws with visible but mild lesions, we felt it important that this was not the case in a RCT. The use of blocks as part of treatment also necessitated an additional crushrestraint intervention to remove blocks from treatment groups that had received them. We considered this necessary first to blind treatment group from the outcome observer and second because work suggests that cows alter their gait while walking on blocks (Higginson Cutler, 2012). Workers wishing to undertake studies such as this may wish to consider their selection criteria, case definitions, and study methodology carefully to avoid some of the logistical problems we encountered.

The study of lameness treatment protocols has lagged behind that of similarly important endemic diseases such as mastitis and infertility. In these fields clinical decision making is based on a plethora of research studies that have tested different treatments and identified the most effective protocols. It is incumbent on the industry and research community to find ways of ensuring that more studies such as this are conducted to provide a robust evidence base to support the effective treatments of this prevalent, costly, and painful endemic disease.

\section{CONCLUSIONS}

In the RCT described here, dairy cows with claw horn lesions treated with a therapeutic trim, a foot block on the sound claw, and a 3-d course of the NSAID ketoprofen were most likely to be sound 5 wk after treatment. Our work suggests that cows benefit from NSAID treatment in addition to the common practices of therapeutic trimming and elevation of the diseased claw using a foot block even when they are newly and mildly lame.

\section{ACKNOWLEDGMENTS}

The work reported here was funded by DairyCo (www.dairyco.org.uk, www.ahdb.org.uk), a levy-funded, not-for-profit organization working on behalf of British dairy farmers and a division of the Agriculture and Horticulture Development Board.

\section{REFERENCES}

Berry, S. L., D. H. Read, T. R. Famula, A. Mongini, and D. Döpfer. 2012. Long-term observations on the dynamics of bovine digital dermatitis lesions on a California dairy after topical treatment with lincomycin $\mathrm{HCl}$. Vet. J. 193:654-658. 
Capion, N., S. M. Thamsborg, and C. Enevoldsen. 2008. Prevalence of foot lesions in Danish Holstein cows. Vet. Rec. 163:80-85.

Cramer, G., K. D. Lissemore, C. L. Guard, K. E. Leslie, and D. F. Kelton. 2008. Herd- and cow-level prevalence of foot lesions in Ontario dairy cattle. J. Dairy Sci. 91:3888-3895.

Edmonson, A. J., I. J. Lean, L. D. Weaver, T. Farver, and G. Webster. 1989. A body condition scoring chart for Holstein dairy cows. J. Dairy Sci. 72:68-78.

Higginson Cutler, J. H. 2012. Welfare of dairy cattle: Epidemiological approaches for detection and treatment of lameness. PhD Thesis. Univ. Guelph, Ontario, Canada.

Horseman, S. V., E. J. Roe, J. N. Huxley, N. J. Bell, C. S. Mason, and H. R. Whay. 2014. The use of in-depth interviews to understand the process of treating lame dairy cows from the farmers' perspective. Anim. Welf. 23:157-165.

Horseman, S. V., H. R. Whay, J. N. Huxley, N. J. Bell, and C. S. Mason. 2013. A survey of the on-farm treatment of sole ulcer and white line disease in dairy cattle. Vet. J. 197:461-467.

Huxley, J. N. 2013. Impact of lameness and claw lesions in cows on health and production. Livest. Sci. 156:64-70.

Laven, R. A., K. E. Lawrence, J. F. Weston, K. R. Dowson, and K. J. Stafford. 2008. Assessment of the duration of the pain response associated with lameness in dairy cows, and the influence of treatment. N. Z. Vet. J. 56:210-217.

Leach, K. A., D. A. Tisdall, N. J. Bell, D. C. J. Main, and L. E. Green 2012. The effects of early treatment for hindlimb lameness in dairy cows on four commercial UK farms. Vet. J. 193:626-632.

Lischer, C. J., U. Koller, H. Geyer, C. Mulling, J. Schulze, and P. Ossent. 2002. Effect of therapeutic dietary biotin on the healing of uncomplicated sole ulcers in dairy cattle - A double blinded controlled study. Vet. J. 163:51-60.

O'Connor, A. M., J. M. Sargeant, I. A. Gardner, J. S. Dickson, M. E. Torrence, C. E. Dewey, I. R. Dohoo, R. B. Evans, J. T. Gray, M. Greiner, G. Keefe, S. L. Lefebvre, P. S. Morley, A. Ramirez, W. Sischo, D. R. Smith, K. Snedeker, J. Sofos, M. P. Ward, and R. Wills. 2010. The REFLECT statement: Methods and processes of creating reporting guidelines for randomized controlled trials for livestock and food safety. Prev. Vet. Med. 93:11-18.

Potterton, S. L., N. J. Bell, H. R. Whay, E. A. Berry, O. C. D. Atkinson, R. S. Dean, D. C. J. Main, and J. N. Huxley. 2012. A descriptive review of the peer and non-peer reviewed literature on the treatment and prevention of foot lameness in cattle published between 2000 and 2011. Vet. J. 193:612-616.

Pyman, M. F. S. 1997. Comparison of bandaging and elevation of the claw for the treatment of foot lameness in dairy cows. Aust. Vet. J. $75: 132-135$.

Silvestre, A. M., F. Petim-Batista, and J. Colaço. 2006. The accuracy of seven mathematical functions in modeling dairy cattle lactation curves based on test-day records from varying sample schemes. J. Dairy Sci. 89:1813-1821.

Toussaint Raven, E. 2002. Cattle Footcare and Claw Trimming. The Crowood Press, Marlborough, UK.

Whay, H. R., A. E. Waterman, and A. J. F. Webster. 1997. Associations between locomotion, claw lesions and nociceptive threshold in dairy heifers during the peri-partum period. Vet. J. 154:155-161. 